\title{
Feedback Control for Adaptive Live Video Streaming
}

\author{
Luca De Cicco \\ Politecnico di Bari \\ Bari, Italy \\ Idecicco@gmail.com
}

\author{
Saverio Mascolo \\ Politecnico di Bari \\ Bari, Italy \\ mascolo@poliba.it
}

\author{
Vittorio Palmisano \\ Politecnico di Bari \\ Bari, Italy \\ vpalmisano@gmail.com
}

\begin{abstract}
Multimedia content feeds an ever increasing fraction of the Internet traffic. Video streaming is one of the most important applications driving this trend. Adaptive video streaming is a relevant advancement with respect to classic progressive download streaming such as the one employed by YouTube. It consists in dynamically adapting the content bitrate in order to provide the maximum Quality of Experience, given the current available bandwidth, while ensuring a continuous reproduction. In this paper we propose a Quality Adaptation Controller (QAC) for live adaptive video streaming designed by employing feedback control theory. An experimental comparison with Akamai adaptive video streaming has been carried out. We have found the following main results: 1) QAC is able to throttle the video quality to match the available bandwidth with a transient of less than 30s while ensuring a continuous video reproduction; 2) QAC fairly shares the available bandwidth both in the cases of a concurrent TCP greedy connection or a concurrent video streaming flow; 3) Akamai underutilizes the available bandwidth due to the conservativeness of its heuristic algorithm; moreover, when abrupt available bandwidth reductions occur, the video reproduction is affected by interruptions.
\end{abstract}

\section{Categories and Subject Descriptors}

C.2.5 [Local and Wide-Area Networks]: Internet; H.5.1 [Multimedia Information Systems]: Video

\section{General Terms}

Design, Performance, Experimentation

\section{Keywords}

Adaptive Video Streaming, quality feedback control, quality adaptation controller

Permission to make digital or hard copies of all or part of this work for personal or classroom use is granted without fee provided that copies are not made or distributed for profit or commercial advantage and that copies bear this notice and the full citation on the first page. To copy otherwise, to republish, to post on servers or to redistribute to lists, requires prior specific permission and/or a fee.

MMSys'11, February 23-25, 2011, San Jose, California, USA.

Copyright 2011 ACM 978-1-4503-0517-4/11/02 ...\$5.00.

\section{INTRODUCTION}

Nowadays, the wide availability of wired and wireless broadband connections is enabling ubiquitous multimedia applications over the Internet, such as video streaming, personal video broadcasting, IPTV, and videoconferencing, at video resolutions that can scale up to full high definition (full HD, 1920x1080) at frame rates up to $30 \mathrm{fps}$. Such rich video contents require a compressed bitstream in the order of 10 Mbps along with adequate processing resources at the client for decoding. Nevertheless, the Internet is becoming more and more accessible to a wide spectrum of devices: if desktops users are normally equipped with large screens, good processing resources, and wired broadband connections, mobile users typically use small screens devices, with limited processing resources and wireless cellular connections that are characterized by variable link characteristics.

Thus, a key challenge is to provide the user with a seamless multimedia experience at the maximum Quality of Experience (QoE) that can be obtained given the available device and network resources. To this purpose, multimedia content must be made adaptive. It is important to notice that the adaptation process should account take into account a wide set of variables such as user screen resolution, CPU load, network available bandwidth, power consumption, some of which are time-varying. In this paper we focus on adaptation to network available bandwidth.

Adaptive (live) video streaming represents a relevant advancement wrt classic progressive download streaming such as the one employed by YouTube.

In classic progressive download streaming, the video is delivered as any data file using greedy TCP connections. The video stream is buffered at the receiver for a while before the playing is started so that short-term mismatches between the video bitrate and the available network bandwidth can be absorbed and video interruptions could be mitigated. Nevertheless, if the mismatch persists the buffer could eventually get empty and playback interruptions could occur affecting the user experience.

On the other hand, with adaptive streaming the video source is adapted on-the-fly so that the user can watch videos at the maximum bitrate that is allowed by the timevarying available bandwidth and by the device resources.

In this paper we focus on a particular adaptive streaming approach that is the stream-switching technique: the server encodes the video content at different bitrates and it switches from one video version to another based on client feedbacks such as the measured available bandwidth. This approach is employed by Apple HTTP live streaming, Mi- 
crosoft IIS server, Adobe Dynamic Streaming, Akamai HD Video Streaming, and Move Networks. In particular, we present a Quality Adaptation Controller (QAC), which has been designed using feedback control, to drive stream-switching for adaptive live streaming applications. The advantages of using a control theoretical approach to design the controller as opposed to a heuristic-based design is a cleaner design that can be not only experimentally tested but also mathematically analyzed.

The rest of the paper is organized as follows: Section 2 provides a brief review of the different adaptive streaming algorithms proposed in the literature along with the main features of the adaptive streaming algorithms employed in commercial products; Section 3 summarizes the results obtained by an experimental investigation of Akamai HD Video Streaming; in Section 4 we propose the Quality Adaptation Controller (QAC) and in Section 5 we experimentally compare QAC with the Akamai HD Video Streaming; finally, Section 6 concludes the paper.

\section{RELATED WORKS}

In this Section we provide a review of the relevant literature on adaptive streaming and then we focus on the most known commercial products providing adaptive streaming services.

\subsection{Adaptive streaming techniques}

In the last decade a vast literature on video streaming has been produced. Main topics that have been investigated are: 1) the design of transport protocols specifically tailored for video streaming, 2) adaptation techniques, 3) scalable codecs.

Concerning the first topic, several transport protocols designed for video streaming have been proposed, such as the TCP Friendly Rate Control (TFRC) [7], Real Time Streaming Protocol (RTSP) [14], Microsoft Media Services (MMS), Real Time Messaging Protocol (RTMP) [3]. Some of the mentioned protocols have been employed in commercial products such as RealNetworks, Windows Media Player, Flash Player. Even though TCP has been regarded in the past as inappropriate for the transport of video streaming protocols, recently it is getting a wider acceptance and it is being used with the HTTP. This is mainly due to the following reasons: i) Internet applications are rapidly converging on web browsers; ii) HTTP-based streaming is cheaper to deploy since it employs standard HTTP servers [17]; iii) TCP has built-in NAT traversal functionalities; iv) it is easy to be deployed within Content Delivery Networks (CDN) [17]; v) TCP delivers most part of the Internet traffic and it is able to guarantee the stability of the network by means of an efficient congestion control algorithm [15].

In [16] the authors develop analytic performance models to assess the performance of TCP when used to transport a live video streaming source without the use of quality adaptation. The theoretical results, obtained considering a constant bit rate (CBR) source and supported by an experimental evaluation, suggest that in order to achieve good performance in terms of startup delay and percentage of late packet arrivals, TCP requires a network bandwidth that is roughly two times the video bit rate. It is important to stress that such bandwidth over-provisioning would systematically waste half of the available bandwidth.

For what concerns adaptation techniques, different ap- proaches have been proposed in the literature so far. The issue here is how to automatically throttle the video quality to match the available resources (network bandwidth, CPU) so that the user receives the video at the maximum possible quality. The proposed techniques to adapt the video source bitrate to the variable bandwidth can be classified into three main categories: 1) transcoding-based, 2) scalable encodingbased, 3) stream-switching (or multiple-bitrate - MBR). Figure 1 shows a schematic representation of each considered technique. In the figure, the blocks represented in gray are those requiring on-the-fly per-client processing and the $(k)$ index refers to variables pertaining to the $k$-th client accessing the same video content. In particular, encoders can be considered as the most CPU-consuming function, whereas controllers generally require much less processing capacity.

The transcoding-based [12] approach (see Figure 1(a)), consists in adapting the video content to match a specific bitrate by means of on-the-fly transcoding of the raw content. These algorithms can achieve a very fine granularity by throttling frame rate, compression, and video resolution. Nevertheless, this comes at the cost of increased processing load and poor scalability, due to the fact that transcoding has to be done on a per-client basis. Another important issue is that such algorithms are difficult to be deployed in CDNs.

Another important class of adaptation algorithms (see Figure 1(b)) employs scalable codecs such as H264/MPEG-4 AVC $[9,10]$. Both spatial and temporal scalability can be exploited to adapt picture resolution and frame rate without having to re-encode the raw video content. With respect to transcoding-based approach, scalable codecs reduce processing costs since the raw video is encoded once and adapted on-the-fly by exploiting the scalability features of the encoder. To be used with CDNs, this approach requires specialized servers implementing the adaptation logic. Also this approach is difficult to be used with CDNs since the adaptation logic requires to be run on specialized servers and content cannot be cached in standard proxies. Another issue is that the adaptation logic depends on the employed codec, thus restricting the content provider to use only a limited set of codecs.

Stream-switching algorithms (see Figure 1(c)) encode the raw video content at increasing bitrates resulting into $N$ versions, i.e. video levels; an algorithm dynamically chooses the video level that matches the user's available bandwidth; those algorithms minimize the processing costs since, once the video is encoded, no further processing is required in order to adapt the video to the variable bandwidth $[17,1$, $11,2,8]$. Another important advantage of such algorithms is that they do not rely on particular functionalities of the employed codec and thus can be made codec-agnostic. The disadvantages of this approach are the increased storage requirements and the fact that adaptation is characterized by a coarser granularity since video bitrates can only belong to a discrete set of levels.

\subsection{Stream-switching adaptive video stream- ing commercial products}

Stream-switching, or Multiple Bit-Rate (MBR) streaming, is gaining momentum since leading commercial media players are preferring it to the other streaming approaches.

IIS Smooth Streaming [17] is a live adaptive streaming service provided by Microsoft. The streaming technology is 


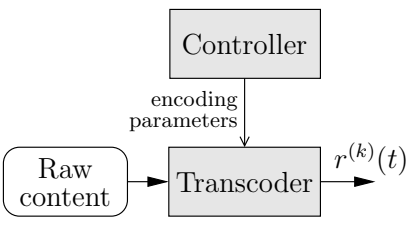

(a) Transcoding-based

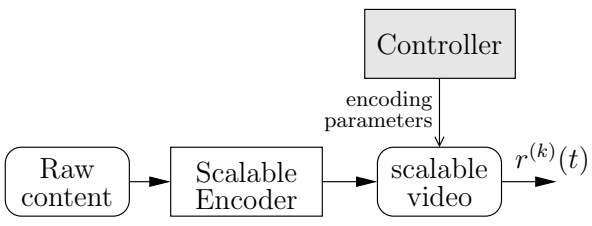

(b) Scalable encoding

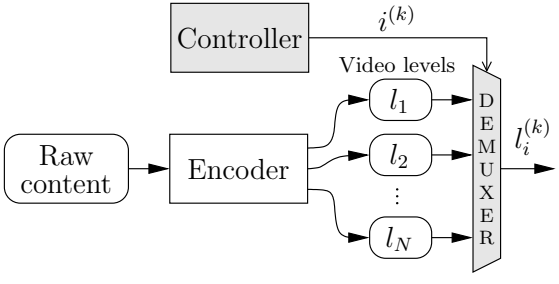

(c) Stream-switching

Figure 1: Adaptive streaming techniques

offered as a web-based solution requiring the installation of a plug-in that is available for Windows and iPhone OS 3.0. IIS Smooth Streaming is codec agnostic and employs a streamswitching approach where different video versions can be encoded with configurable bitrates and video resolutions up to $1080 \mathrm{p}$. In the default configuration, the video is encoded in seven layers ranging from $300 \mathrm{kbps}$ up to $2.4 \mathrm{Mbps}$.

Adobe Dynamic Streaming [8] is a web-based adaptive streaming service developed by Adobe that is available to all devices running a browser with Adobe Flash plug-in. The server stores several streams at different quality and resolution and switches among them during the playback, in order to match user bandwidth and CPU. The service is provided using the RTMP streaming protocol [3]. The supported video codecs are H.264 and VP6, which are included in the Adobe Flash plug-in.

Apple has recently released a client-side HTTP Adaptive Live Streaming solution [11]. The server segments the video content into several pieces with configurable duration and video quality. The server exposes a playlist (.m3u8) containing all the available video segments. The client downloads consecutive video segments and it dynamically chooses the video quality by using an undisclosed algorithm. Apple HTTP Live Streaming employs H.264 codec using a MPEG2 TS container and it is available on any device running iPhone OS 3.0 or later (including iPad), or any computer with QuickTime X or later installed.

Move Networks provides live adaptive streaming service to several TV networks such as ABC, FOX, Televisa, ESPN and others. A plug-in, available for the most used web browsers (Windows and Mac OS X) has to be installed to access the service. Move Networks employs VP7, a video codec developed by On2, a company that has been recently acquired by Google. Adaptivity to available bandwidth is provided using the stream-switching approach. Five different versions of the same video are available at the server with bitrates ranging from $100 \mathrm{kbps}$ up to $2200 \mathrm{kbps}$.

$H u l u^{1}$ offers on demand TV shows and movies in the USA. In 2010 Hulu has launched a new video player that implements adaptivity by employing the stream-switching approach. The adaptation algorithm does not change the video frame rate, whereas it sets the video resolution to match the current user available bandwidth.

\section{AKAMAI ADAPTIVE STREAMING}

In this Section we summarize and significantly extend the results obtained in a recent experimental investigation of the Akamai HD Video Streaming (AHDVS) service [5].

\footnotetext{
${ }^{1}$ http://www.hulu.com
}

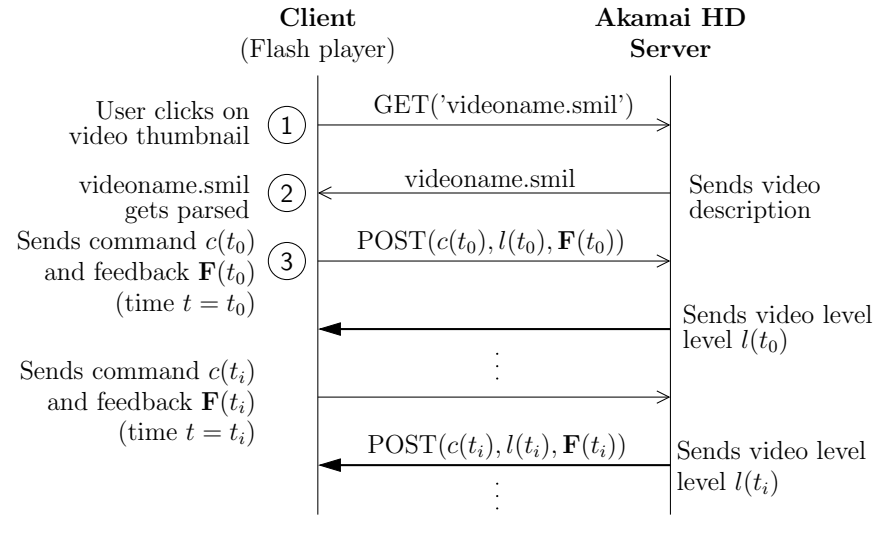

Figure 2: Client-server time sequence graph: thick lines represent video data transfer, thin lines represent HTTP requests sent from client to server

\subsection{Client-server protocol}

AHDVS employs HTTP connections to stream data from the server to the client. The adaptation algorithm is executed at the client in a Flash application. By analyzing the traffic between the Akamai server and the client we have observed that the client issues a number of HTTP requests to the server throughout all the duration of the video streaming. Figure 2 shows a typical time sequence graph of the HTTP requests sent from the client to the Akamai server.

At first, the client connects to the Akamai server [1], then a Flash application is loaded and a number of videos are made available to the client. When the user clicks on the thumbnail (1) of the video he is willing to play, a GET HTTP request is sent to the server which points to a SMIL ${ }^{2}$ compliant file. In the SMIL file the base URL of the video, the available video levels, and the corresponding encoding bit-rates are provided.

After that, the client parses the SMIL file (2) to reconstruct the complete URLs of the available video levels and selects the corresponding video level based on the quality adaptation algorithm. All the videos available on the demo website are encoded at five different bitrates as shown in Table 1. In particular, the video level bitrate $l(t)$ can assume values in the discrete set of available video levels $\mathscr{L}=\left\{l_{0}, \ldots, l_{4}\right\}$. Video levels are encoded at 30 frames per second (fps) using H.264 codec with a group of picture (GOP) of length 36, so that two consecutive I frames are $1.2 \mathrm{~s}$ apart. This means that, since a video switch can oc-

\footnotetext{
$\overline{2}$ http://www.w3.org/TR/2005/REC-SMIL2-20050107/
} 


\begin{tabular}{|c|c|c|}
\hline $\begin{array}{c}\text { Video } \\
\text { level }\end{array}$ & $\begin{array}{c}\text { Bitrate } \\
\text { (kbps) }\end{array}$ & $\begin{array}{c}\text { Resolution } \\
(\text { width } \times \text { height) }\end{array}$ \\
\hline \hline$l_{0}$ & 300 & $320 \times 180$ \\
\hline$l_{1}$ & 700 & $640 \times 360$ \\
\hline$l_{2}$ & 1500 & $640 \times 360$ \\
\hline$l_{3}$ & 2500 & $1280 \times 720$ \\
\hline$l_{4}$ & 3500 & $1280 \times 720$ \\
\hline
\end{tabular}

Table 1: Set of available video levels $\mathscr{L}$

\begin{tabular}{|l|l|c|c|}
\hline & Command & Args & Occurrence (\%) \\
\hline \hline$c_{1}$ & throttle & 1 & $\sim 80 \%$ \\
\hline$c_{2}$ & rtt-test & 0 & $\sim 15 \%$ \\
\hline$c_{3}$ & SWITCH_UP & 5 & $\sim 2 \%$ \\
\hline$c_{4}$ & BUFFER_FAILURE & 7 & $\sim 2 \%$ \\
\hline$c_{5}$ & log & 2 & $\sim 1 \%$ \\
\hline
\end{tabular}

Table 2: Commands issued by the client to the streaming server via the cmd parameter

cur only at the beginning of a GOP, video levels can change only each 1.2s. Finally, the audio is encoded with Advanced Audio Coding (AAC) at $128 \mathrm{kbps}$ bitrate.

After the SMIL file gets parsed, at time $t=t_{0}(3)$, the client issues the first POST request specifying several parameters. Among those, the most important parameters are cmd, that specifies a command the client issues on the server, and lvl1, that specifies several feedback variables $\mathbf{F}(t)$ such as: 1) the receiver buffer size $q(t), 2)$ the receiver buffer target $\left.q_{T}(t), 3\right)$ the received video frame rate $\left.f(t), 4\right)$ the estimated bandwidth $B(t), 5)$ the received goodput $r(t)$, 6 ) the current received video level bitrate $l(t)$.

At time $t=t_{0}$, the quality adaptation algorithm starts. For a generic time instant $t_{i}>t_{0}$ the client issues commands via HTTP POST requests to the server in order to select the suitable video level. It is worth to notice that the commands are issued on a separate TCP connection that is established at time $t=t_{0}$.

Table 2 reports the possible commands $c_{i}$ that the client can issue on the servers along with the number of arguments and the occurrence percentage. The first two commands are issued periodically, throttle with a median interdeparture time of about $2 \mathrm{~s}$ and rtt-test with a median inter-departure time of about 11s. On the other hand, log, SWITCH_UP and BUFFER_FAILURE are commands triggered on the occurrence of a particular event.

In [5] we have shown that the throttle command specifies a single argument, the throttle percentage $T(t)$, that it is used to control the receiver buffer level $q(t)$ as we will discuss in Section 3.2. The rtt-test command is issued to periodically actively probe for the available bandwidth and the round trip time $R(t)$ (RTT) of the connection.

Finally, the two event-based commands SWITCH_UP and BUFFER_FAILURE are sent from the client to ask the server to respectively switch up or down the video level $l(t)$.

\subsection{The control system}

Figure 3 shows a block diagram of the control architecture employed by AHDVS. The server is connected to the client through an Internet connection characterized by a forward connection delay $\tau_{f}$ and a backward connection delay $\tau_{b}$. Figure 3 shows that the three main components of the

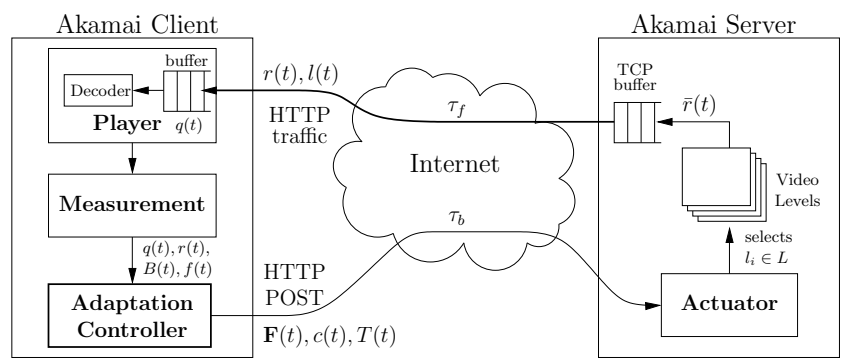

Figure 3: A block diagram of the control architecture employed by AHDVS

control loop, i.e. measurement, adaptation controller, and actuator, are connected through the Internet so that the control loop is affected by an overall delay $\tau=\tau_{f}+\tau_{b}$.

The client receives the video flow at level $l(t) \in \mathscr{L}$ over an HTTP connection at a rate $r(t)$. The received video is stored in a playout buffer, whose instantaneous length is $q(t)$, which is drained by the decoder at the current received video level $l(t)$. A measurement module feeds the values of the buffer length $q(t)$, the received goodput $r(t)$, the bandwidth $B(t)$, and the decoded frame rate $f(t)$ to the adaptation controller.

The adaptation controller is made of two modules: 1) a playout buffer level controller whose goal is to drive the buffer length to a target length; 2) a stream-switching logic that selects the appropriate video level to be streamed by the server.

In [5] we have shown that the control law implemented by Akamai to regulate the buffer length $q(t)$ is a proportional controller that takes the error $q_{T}(t)-q(t)$ as the input and whose output is the throttle percentage $T(t)$ :

$$
T(t)=\max \left(\left(1+\frac{q_{T}(t)-q(t)}{q_{T}(t)}\right) 100,10\right)
$$

The throttle percentage $T(t)$ is used to set the rate $\bar{r}(t)$ at which the Akamai server feeds the TCP socket buffer with the current video level $l(t)$ as follows:

$$
\bar{r}(t)=l(t) \frac{T(t)}{100}
$$

The rationale of controlling $\bar{r}(t)$ is to induce, on average, a TCP sending rate that is equal to $\bar{r}(t)$. This means that when the throttle percentage is above $100 \%$ the server can stream the video at a rate that is above the encoding bitrate $l(t)$. It is important to stress that, in the case of live streaming, it is not possible for the server to supply a video at a rate that is above the encoding bitrate for a long period, since the video source is not pre-encoded.

By looking at (1) we find that when the buffer length $q(t)$ matches the target buffer length $q_{T}(t)$, the throttle percentage $T(t)$ is equal to $100 \%$ and $\bar{r}(t)$ matches $l(t)$. On the other hand, when the error $q_{T}(t)-q(t)$ increases, $T(t)$ increases accordingly in order to allow $\bar{r}(t)$ to increase so that the buffer can be filled quickly. Since (1) implements a simple proportional controller on the buffer length, the $q(t)$ matches $q_{T}(t)$ with an offset at steady state [6].

Let us now focus on the stream-switching logic that is a heuristic-based controller that decides which video level $l(t) \in \mathscr{L}$ has to be sent by the server, based on the estimated bandwidth, the current video level, the playout buffer 


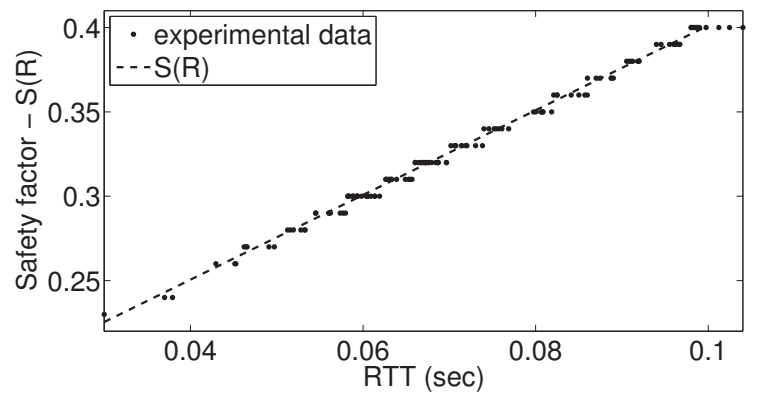

Figure 4: Safety factor vs round trip time.

length, and the frame rate. In particular, and based on the debug information provided by the Akamai Client and on the experiments we have run, the stream-switching heuristic works as follows.

The client periodically issues rtt-test commands that have the effect of setting at the server a throttling percentage of $500 \%$, thus asking the server to periodically send the video in greedy mode. In this way Akamai actively probes for extra available bandwidth and estimates the RTT $R(t)$ under congestion. Based on the estimated value of the RTT, the client computes a safety factor $S$. By parsing the debug information in order to collect the pairs $(R(t), S(t))$ shown in Figure 4, it was possible to run a linear regression over the dataset which yielded to the following static linear model $(R(t)$ is expressed in seconds):

$$
f(R(t))=2.5 R(t)+0.15
$$

We have observed that when $R(t)>0.1$ s the safety factor remains set to 0.4 , whereas when $R(t)<0.02 \mathrm{~s}$, it is set to 0.2 . Thus, we can conclude that the complete model for $S(R(t))$ is the following:

$$
S(R(t))= \begin{cases}0.2 & 0<R(t)<0.02 \mathrm{~s} \\ 2.5 R(t)+0.15 & 0.02 \mathrm{~s} \leq R(t) \leq 0.1 \mathrm{~s} \\ 0.4 & R(t)>0.1 \mathrm{~s}\end{cases}
$$

For each video level $l_{i} \in \mathscr{L}$ a high threshold $L_{i}^{H}$ and a low threshold $L_{i}^{L}$ are maintained:

$$
L_{i}^{H}(t)=l_{i} \cdot(1+S(t)) ; L_{i}^{L}=l_{i} \cdot 1.2
$$

A switch up (SWITCH_UP) to a higher video level $l_{i}$ is enabled only if $B(t)>L_{i}^{H}(t)$, which means that if, for instance, the RTT is above $0.1 \mathrm{~s}$ and thus $S(R(t))=0.4$, in order to switch to level $l_{i}$ the estimated bandwidth must be at least $40 \%$ higher than $l_{i}$. This seems to be a conservative approach that leads to network underutilization and, as a consequnece, to a reduced QoE.

The switch down event occurs when:

$$
q(t)<q_{L}(t)
$$

where $q_{L}(t)$ is another threshold that is smaller than the queue target ${ }^{3} q_{T}(t)$. When (5) holds, a BUFFER_FAILURE is sent and the new video level $l_{i}<l(t)$ is selected. In particular, the highest video level $l_{i} \in \mathscr{L}$ satisfying the following condition:

$$
B(t)>1.2 \cdot l_{i}=L_{i}^{L}
$$

\footnotetext{
${ }^{3}$ The identification of $q_{L}(t)$ has not been carried out.
}

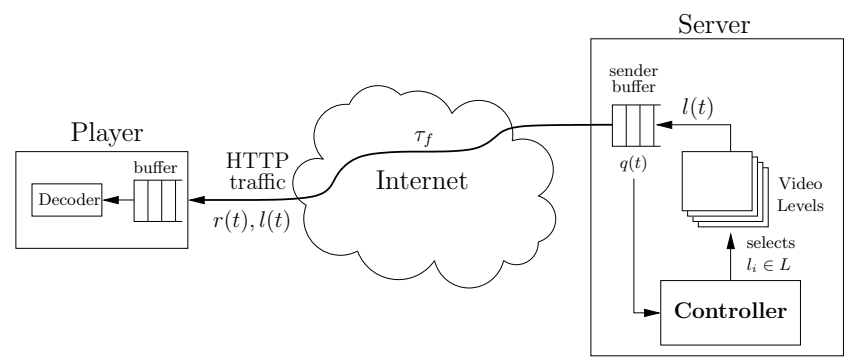

Figure 5: QAC control architecture

is selected. Thus, in to select the level $l_{i}$, the currently estimated bandwidth $B(t)$ must be at least $20 \%$ above $l_{i}$. Moreover, in [5] we have shown that when SWITCH_UP and BUFFER_FAILURE commands are sent from the client, the actuator, which is located at the server, takes a delay of $\tau_{s u} \simeq 14 \mathrm{~s}$ and $\tau_{s d} \simeq 7 \mathrm{~s}$ respectively, to actuate these commands.

Finally, it is worth noting that the overall system exhibits a very complex dynamics due to the interaction of two closed-loop dynamics: the stream-switching logic, which has been designed using heuristic arguments, and the buffer level controller. As a consequnce, it is very complex to develop a mathematical analysis as well as to tune control variables to satisfy key design requirements such as settling times and steady state errors.

\section{QUALITY ADAPTATION CONTROLLER}

In this Section we propose a Quality Adaptation Controller (QAC) for adaptive live video streaming that aims at pursuing the following goals: 1) maximize the $Q o E$ by delivering the best quality that is possible given the network available bandwidth while minimizing playback interruptions; 2) rigorous design of the controller by employing the control theory; 3) high scalability in terms of processing costs; 4) $C D N$-friendly design, i.e. the algorithm can be easily deployed on CDNs; 5) codec-agnostic, i.e. the service provider has the freedom to choose any codec.

In order to pursue the goals 3), 4), and 5) we choose the stream-switching approach and we employ the standard HTTP streaming over TCP. For what concerns the goals 1) and 2) we employ feedback control theory to design a controller that throttles the video level $l(t)$ to be streamed without using any heuristics. This provides the key advantage of getting a predictable system dynamics that can fulfill required design features such as settling time and steady state errors [6].

\subsection{The control system}

Figure 5 shows the architecture of the proposed streaming server. The first important difference wrt the control architecture employed by Akamai (Figure 3) is that measuring, control and actuation take place at the server so that the control loop is not affected by delays and does not require explicit feedback from the client. This architecture provides the following advantages: 1) simplicity of the player: being the control centralized at the server, the player at the client has the only task of decoding and playing the stream; moreover, when a new version of the control algorithm is designed and installed at the server, there is no need to update the 


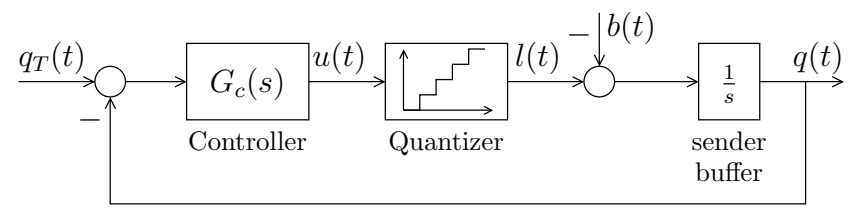

Figure 6: Block diagram of the control loop

player; 2) effectiveness of the controller: by avoiding delays in the control loop the controller can provide faster dynamics while retaining stability [6].

The controller works as follows: it takes as input the queue length $q(t)$ of the sender buffer that is placed at the server, and it selects the video level $l_{i} \in \mathscr{L}$. The selected video level is temporally stored at the sender buffer and is then sent to the client via a TCP connection. The received stream is buffered at the client that decodes and plays the video content.

Figure 6 shows a block diagram of the feedback control system designed to throttle the video level $l(t)$. In the following $s \in \mathbb{C}$ denotes the Laplace variable and $F(s)=\mathcal{L}\{f(t)\}$ denotes the unilateral Laplace transform of the real valued function $f(t)$.

The input of the system $q_{T}$ is the set-point, or threshold value, for the sender buffer length $q(t)$.The controller goal is to track a queue length $q_{T}>0$ so that the TCP sender buffer is always full and can fill the communication pipe.

The controller, which can be described by its transfer function $G_{c}(s)$, takes as input the error $e(t)=q_{T}-q(t)$ and outputs the control signal $u(t)$ that is the bitrate the encoder should set to match the available bandwidth $b(t)$. In our case, since we employ the stream-switching approach, the video bitrate will belong to the discrete set of available video levels $\mathscr{L}$. This can be modelled through a quantizer, which is a static element that takes as input $u(t)$ and selects the highest video level $l_{i}$ that is less then $u(t)$. Finally, the sender buffer, which can be modelled by the integrator $1 / s$, is filled at a rate $l(t)$ and it is drained by the available bandwidth at the rate $b(t)$. It is worth to notice that the available bandwidth $b(t)$ is modelled as a disturbance [13].

The effect of the quantizer is to add a quantization error $d_{q}(t)=l(t)-u(t)$ to $u(t)$. This is equivalent to consider $d_{q}(t)$ as a disturbance acting on $b(t)$ giving the total equivalent disturbance $d_{e q}(t)=b(t)+d_{q}(t)$. In this way we are able to take the quantizer out of the control loop and we can compute the transfer function from the input $q_{T}$ to the output $q(t)$ as follows:

$$
G_{0}(s)=\frac{Q(s)}{Q_{T}(s)}=\frac{G_{c}(s) \frac{1}{s}}{1+G_{c}(s) \frac{1}{s}}
$$

We choose a proportional integral (PI) controller:

$$
G_{c}(s)=\frac{U(s)}{E(s)}=K_{p}+\frac{K_{i}}{s}
$$

since it is able to reject step-like disturbances $b(t)$ and it is very simple to be discretized and implemented in a software module. The integral action of the controller ensures that the video level $l(t)$ matches on average the available bandwidth $b(t)$

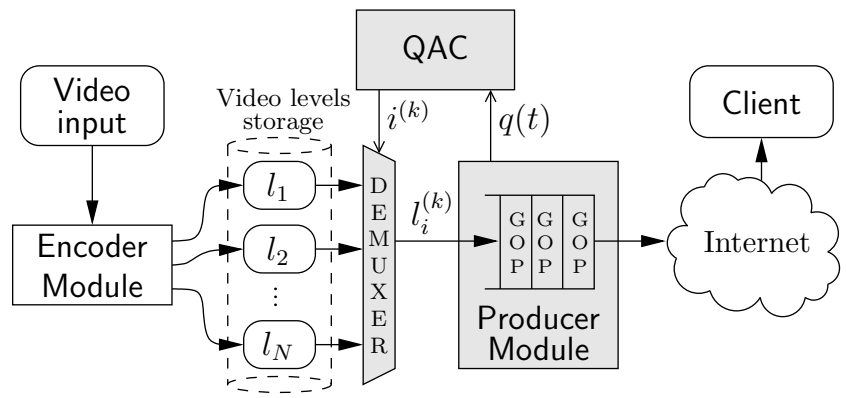

Figure 7: The QAC adaptive streaming server architecture

By substituting (7) in (6) it turns out:

$$
G_{0}(s)=\frac{K_{p} s+K_{i}}{s^{2}+K_{p} s+K_{i}}
$$

Thus, the closed loop system is a second order system with one zero. In order to tune the controller, we impose the damping factor of the system (8) to be $\delta=\sqrt{2} / 2[6]$ and a natural frequency $\omega_{n}=\sqrt{K_{i}}=0.1886 \frac{\mathrm{rad}}{\mathrm{s}}$ that corresponds to a system bandwidth of around $0.06 \mathrm{~Hz}$ and a $2 \%$ settling time of $T_{s}=\frac{4}{\delta \omega_{n}}=30 \mathrm{~s}$. This choice is made in order to limit the switching frequency between different video levels. The gains of the PI turn out to be $K_{i}=0.0356$ and $K_{p}=$ 0.2667 .

In the time domain the control law is:

$$
u(t)=\mathcal{L}^{-1}\left\{G_{c}(s) E(s)\right\}=K_{p} e(t)+K_{i} \int_{0}^{t} e(\xi) d \xi
$$

In order to implement (9) we need to discretize the control law with a sampling time $\Delta T$ :

$$
u\left(t_{k}\right)=K_{p} e\left(t_{k}\right)+K_{i} \sum_{j=0}^{k} \Delta T e\left(t_{j}\right)
$$

We choose a sampling time $\Delta T=0.5 \mathrm{~s}$ that is $1 / 60$ th of the settling time $T_{s}$. In the following subsection we provide the implementation details of the adaptive streaming server using the QAC.

\subsection{Implementation of the adaptive streaming server}

The adaptive streaming server is written in Python and developed using the Twisted ${ }^{4}$ libraries. A schematic representation of the proposed streaming server is shown in Figure 7. The server contains an audio/video transcoding engine (Encoder Module) developed using GStreamer ${ }^{5}$ and FFMpeg ${ }^{6}$ libraries. The encoder module takes as input a raw or pre-encoded audio/video file and outputs a set of files transcoded at various bitrates and resolutions. We used the same levels of AHDVS as shown in Table 1 with a frame rate equal to $30 \mathrm{fps}$. We employ a fixed Group of Picture (GOP) of 30 frames which is equal to 1 s of video stream. For each transcoded file, the encoder module stores an index file (.index) containing the file position and the timestamp of each encoded GOP. We used a fixed GOP

\footnotetext{
${ }^{4}$ http://twistedmatrix.com/

${ }^{5}$ http://gstreamer.org/

${ }^{6}$ http://www.ffmpeg.org/
} 


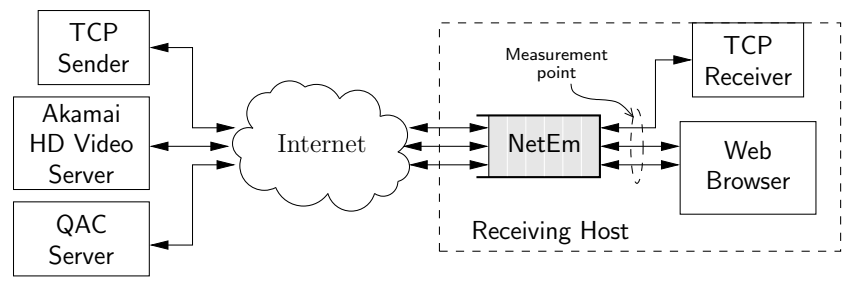

Figure 8: Testbed employed in the experimental evaluation

encoder setting in order to simplify the stream switch between video levels. Moreover, the server integrates also a Producer Module, which is a simple HTTP server. When a client connects to the server, it sends a GET HTTP request specifying the stream unique identifier it wants to play. The producer replies with a HTTP response and starts to send the video stream content reading from the storage at a configured start level ${ }^{7} l(0)=\bar{l}$. Moreover, the producer continuously provides the current queue level $q(t)$ to the QAC module. When a video level switch occurs, the producer selects the corresponding input file from the storage, it performs a file seek operation to the current sent time position using the information contained in the .index file and then it feeds the data to the client. The switch operation can be performed only at GOP boundaries in order to ensure the correct decoding by the client.

The adaptive streaming server supports every encoding format provided by GStreamer/FFMpeg libraries. In this paper, in order to make a fair comparison with AHDVS, we encoded the video using H.264 codec and MP3 audio muxed into FLV container.

It is worth noticing that the producer and the QAC modules are independent from the encoding profile used. Finally, we stress that the client can be not only an Adobe Flash applet, but also any video player that supports the same codec employed by the server. A buffering time of $15 \mathrm{~s}$ at the client side is recommended in order to avoid interruptions.

\section{EXPERIMENTAL EVALUATION}

In this section we carry out a comparison between the Akamai HD video server and the proposed Quality Adaptation Controller (QAC) by employing the testbed shown in Figure 8. To run the experiments, we have employed the video sequence "Elephant's Dream" 8 since its duration is long enough for a careful experimental evaluation. In order to perform a fair comparison, the video sequence streamed with the QAC has been encoded using the $\mathrm{x} 264$ codec and the same discrete set of video levels employed by AHDVS (see Table 1). The receiving host is an Ubuntu Linux machine running 2.6.32 kernel equipped with NetEm, which is a kernel module that, along with the traffic control tools available on Linux kernel, allows downlink channel bandwidth and delays to be set. In order to perform traffic shaping on the downlink we have used the Intermediate Functional Block pseudo-device IFB $^{9}$.

The receiving host was connected to the Internet through our campus wired connection. It is worth to notice that,

\footnotetext{
${ }^{7}$ In this paper we used a start video level $l(0)=l_{1}$

${ }^{8}$ http://orange.blender.org/

${ }^{9}$ http://linuxfoundation.org/collaborate/workgroups/networking/ifb
}

before running any experiment, we carefully checked that the available bandwidth was well above $4 \mathrm{Mbps}$, which is the maximum value of the bandwidth we set in the traffic shaper. The measured RTT between our client and the Akamai server was in the range $10 \mathrm{~ms}$ to $30 \mathrm{~ms}$. All measurements have been taken after the traffic shaper (as shown in Figure 8 ) and collected by sniffing the traffic on the receiving host with tcpdump. For what concerns AHDVS, the dump files have been post-processed and parsed using a Python script to obtain the figures that we report in the following.

The receiving host runs an iperf server (TCP Receiver) in order to receive TCP greedy flows sent by an iperf client (TCP Sender).

Four different scenarios have been considered in order to investigate the dynamic behaviour of the two considered quality adaptation algorithms: 1) one video stream over a bottleneck link whose available bandwidth changes following a step function with minimum value of $500 \mathrm{kbps}$ and maximum value of $4000 \mathrm{kbps}$; 2) one video stream over a bottleneck link whose available bandwidth varies as a square wave with a period of $200 \mathrm{~s}$, a minimum value of $500 \mathrm{kbps}$ and a maximum value of $4000 \mathrm{kbps}$; 3) one video stream sharing a bottleneck, whose available bandwidth is equal to $4000 \mathrm{kbps}$, with one concurrent TCP flow; 4) two video streams sharing a bottleneck whose available bandwidth is equal to $4000 \mathrm{kbps}$.

In scenarios 1 and 2 abrupt variations of the available bandwidth occur: such step-like variations of the input signal are often employed in control theory to evaluate key features of a dynamic system response to an external input such as settling time, overshoots and time constants [4]. The third scenario evaluates the dynamic behaviour of a video flow when it shares the bottleneck with a greedy TCP flow, such as in the case of a file download, and it is useful to investigate the inter-protocol fairness.

Since, due to the use of TCP, the loss rate is small, the evaluation of the QoE can be inferred by evaluating the instantaneous video level received by the client, i.e., the higher the received video level $l(t)$ the higher the quality perceived by the user. For this reason we employ the received video level $l(t)$ as the key performance index of the system. In particular, to assess the efficiency of the quality adaptation algorithm, we introduce the following index of utilization:

$$
\eta=\frac{\hat{l}}{C}
$$

where $\hat{l}$ is the average value of the video level $l(t), C=$ $\min \left(l_{M}, b\right)$ where $l_{M}$ is the maximum video level and $b$ is the available bandwidth. The index $0 \leq \eta \leq 1$ is 1 when the average value of the received video level is equal to $C$, i.e. when the video level exactly matches the bottleneck available bandwidth.

For each considered scenario we will show the dynamics of the following variables: the received video level $l(t)$, the received video rate $r(t)$, the decoded frame rate $f(t)$, and the receiver buffer length $q(t)$.

\subsection{Step-like change of the bottleneck capacity}

We start by investigating the dynamic behaviour of the two quality adaptation algorithms in a simple scenario. The bottleneck available bandwidth $b(t)$ increases at time $t=$ $50 \mathrm{~s}$ from a value of $A_{m}=500 \mathrm{kbps}$ to a value of $A_{M}=$ $4000 \mathrm{kbps}$. It is worth to notice that $A_{m}>l_{0}$ and $A_{M}>l_{4}$. 


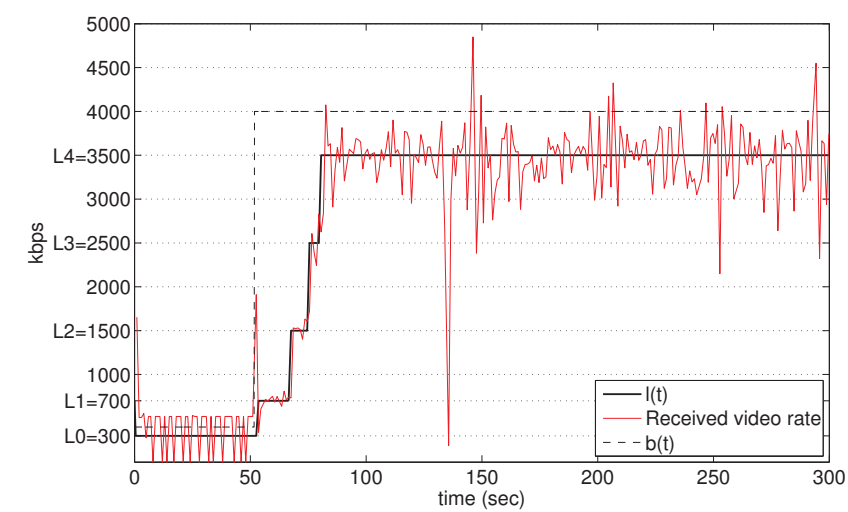

(a) Received rate $r(t)$, video level $l(t)$, and available bandwidth $b(t)$

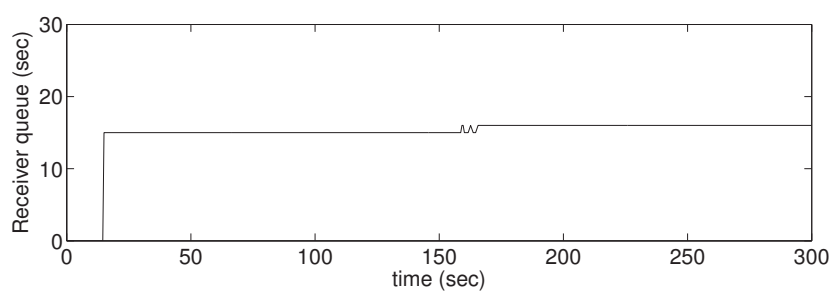

(b) Receiver buffer length

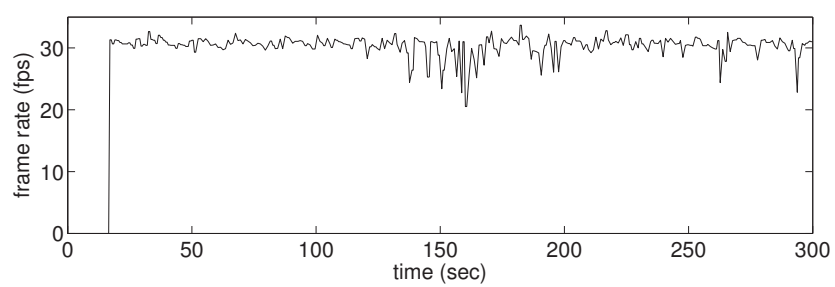

(c) Frame rate $f(t)$

Figure 9: QAC adaptive video streaming response to a step change of available bandwidth at $t=50 \mathrm{~s}$

In particular, we are interested in assessing the responsiveness of the adaptation algorithms in matching the available bandwidth choosing the adequate video level $l(t)$. Figure 9 and Figure 10 show the dynamics of one QAC and one AHDVS video flow, respectively.

Let us consider Figure 9(a) that shows the received video rate $r(t)$ and video level $l(t)$ in the case of QAC: after that the bandwidth increases at $t=50 \mathrm{~s}$, the video level increases and eventually reaches, at steady state, the maximum video level $l_{4}$ after a transient time of around 30s. It is worth noting that the transient time required for $l(t)$ to match the available bandwidth $b(t)$ is equal to the settling time $T_{s}$ that was set as requirement when the quality controller was designed (7) (see Section 4). Moreover, Figure 9(b) shows that the received buffer length is $15 \mathrm{~s}$ throughout all the duration of the connection. The decoded frame rate of the stream oscillates around $30 \mathrm{fps}$, which proves that there were no video interruptions during the streaming. Finally, the efficiency index (11) is 0.93 .

Let us now focus on the Akamai video streaming server. Figure 10(a) shows the dynamics of the video level $l(t)$, the estimated bandwidth reported by the lvl1 parameter, and

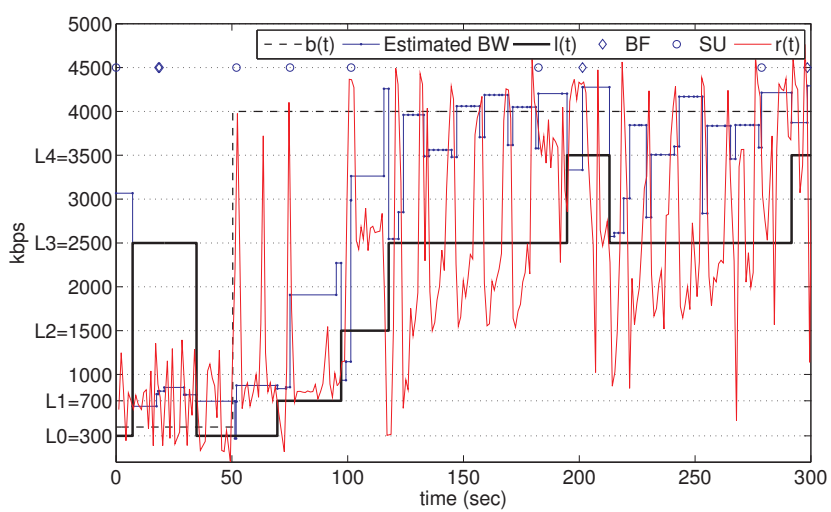

(a) Estimated $\mathrm{BW}$, video level $l(t)$, received rate $r(t)$, BUFFER_FAILURE, and SWITCH_UP events

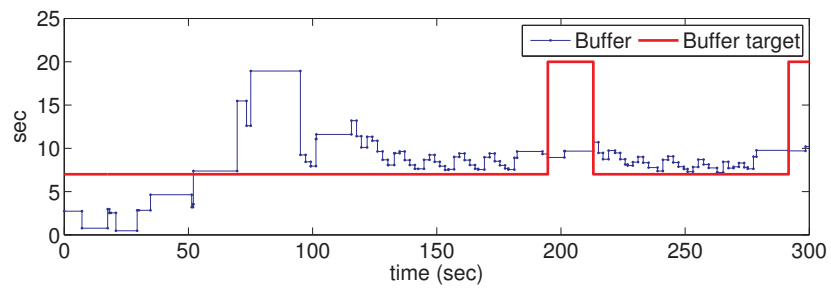

(b) Receiver buffer length and target buffer length

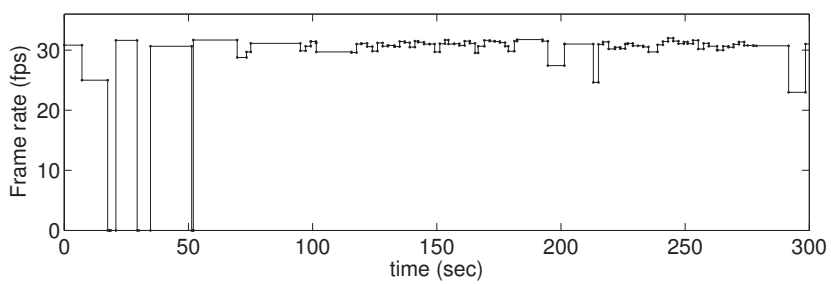

(c) Frame rate $f(t)$

Figure 10: AHDVS response to a step change of available bandwidth at $t=50 \mathrm{~s}$

the received video rate $r(t)$. In order to show their effect on the dynamics of $l(t)$, Figure 10(a) also reports the time instants at which BUFFER_FAILURE (BF) and SWITCH_UP (SU) commands are issued. The video level is initialized at $l_{0}$ that is the lowest available version of the video. Nevertheless, at time $t=0$ the estimated bandwidth is erroneously overestimated to a value above $3000 \mathrm{kbps}$ and a SWITCH_UP command is sent to the server. The effect of this command occurs after an actuation delay of $\tau_{s u}=7.16 \mathrm{~s}$ (see Section 3 ) when $l(t)$ is increased to $l_{3}=2500 \mathrm{kbps}$, which is the video level closest to the bandwidth estimated at $t=0$. By setting the video level to $l_{3}$, which is above the current available bandwidth $A_{m}=500 \mathrm{kbps}$, the receiver buffer starts to drain and it eventually gets empty at $t=17.5 \mathrm{~s}$ (see Figure 10(b)). Figure 10(c) shows that during the time interval $[17.5,20.8] \mathrm{s}$ the playback frame rate is zero, meaning that the video is paused. At time $t=18.32 \mathrm{~s}$, a BUFFER_FAILURE command is finally sent to the server. After a delay of about $\tau_{s d}=16 \mathrm{~s}$ the server switches the video level to $l_{0}=300 \mathrm{kbps}$ that is below the available bandwidth $A_{m}$. Even though the heuristic to trigger a video level switch down (5) should be able in principle to avoid interruptions, the actuation delay $\tau_{s d}$ poses a remarkable limitation to the 
responsiveness of the quality adaptation algorithm. Moreover, Figure 10(a) shows that the transient time required by $l(t)$ to reach the maximum video level $l_{4}$ is around $150 \mathrm{~s}$, which is roughly one order of magnitude higher than the transient time exhibited by QAC. Finally, in this case the efficiency index (11) is 0.676 that is well below the value found in the case of QAC. To conclude, the inefficiency of AHDVS is largely due to the conservativeness of the safetyfactor $S(t)$ that we discussed in Section 3. In fact, given a minimum safety factor of $S=0.2$, the available bandwidth required to switch to the level $l_{4}=3500 \mathrm{kbps}$ according to (4) turns out to be $4200 \mathrm{kbps}$ that is above $A_{M}$.

Let us compare the received video rates of QAC and AHDVS shown respectively in Figure 9(a) and 10(a): if on one hand the received video rate of $\mathrm{QAC}$ is affected by a moderate burstiness that is typical of a TCP connection, on the other hand the received rate of AHDVS is affected by remarkable and persistent oscillations whose amplitude is more than $2 \mathrm{Mbps}$. This is due to the fact that AHDVS dynamics periodically switches between two states: in the normal state the video sending rate is bounded by the maximum sending rate $\bar{r}(t)$ given by (2), whereas each time a rtt-test command is issued AHDVS enters the greedy-mode state and for a short time interval of around $5 \mathrm{~s}$ the sending rate is limited by the available bandwidth [5].

In conclusion, this experiment shows that QAC is able to provide the maximum value of the received video level that is possible given the available bandwidth with a transient time of around 30s in accordance with the design requirements given in Section 4. On the other hand, AHDVS exhibits a very large transient of around 150s, remarkable oscillations in the received rate $r(t)$, it is not able to provide the maximum possible QoE to the user, and it is not able to avoid interruptions.

\subsection{Square-wave varying bottleneck capacity}

In this experiment we consider abrupt drops/increases of the bottleneck available bandwidth $b(t)$ which is shaped as a square-wave function with a period of $200 \mathrm{~s}$, a minimum value $A_{m}=500 \mathrm{kbps}$ and a maximum value $A_{M}=$ $4000 \mathrm{kbps}$. The aim of this experiment is to assess the responsiveness of the two considered adaptive video streaming services in shrinking the video level $l(t)$ in response to an abrupt drop of the available bandwidth and to what extent they are able to guarantee a continuous reproduction of the video content in the presence of this sudden bandwidth reduction.

Figure 11(a) shows the dynamics of the video received rate $r(t)$ and the video level $l(t)$ in response to the available bandwidth $b(t)$. The figure shows that the QAC algorithm is able to control $l(t)$ so that it properly follows step increases and decreases in the available bandwidth. In particular, the transient times required for $l(t)$ to match bandwidth increases/decreases are less than 20s. Moreover, Figures 11(b) and 11(c) show that the receiver buffer length is around $15 \mathrm{~s}$ and the reproduced frame rate is around $30 \mathrm{fps}$ during all the experiment, so showing a reproduction without interruptions. During the time intervals with bandwidth $A_{M}=4000 \mathrm{kbps}$, the efficiency index was equal to 0.93 .

On the other hand, Figure 10 clearly shows that AHDVS is not able to properly adapt the video level to follow bandwidth variations. By considering the dynamics of the video level $l(t)$ shown in Figure 12(a) we notice two main facts:

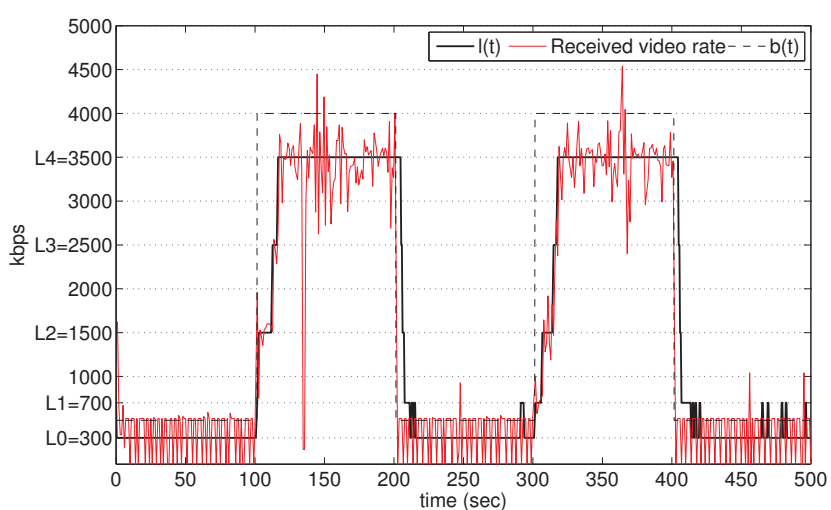

(a) Received rate $r(t)$, video level $l(t)$, and available bandwidth $b(t)$

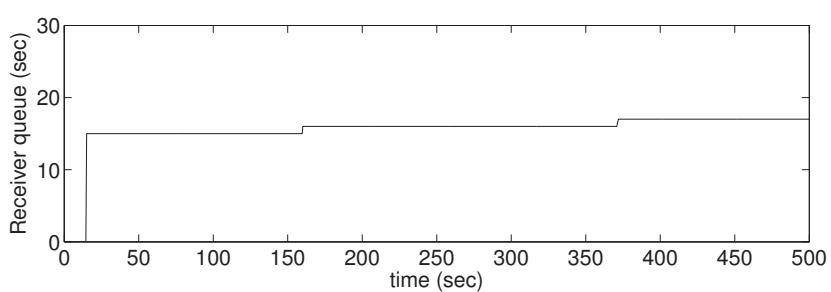

(b) Receiver buffer length

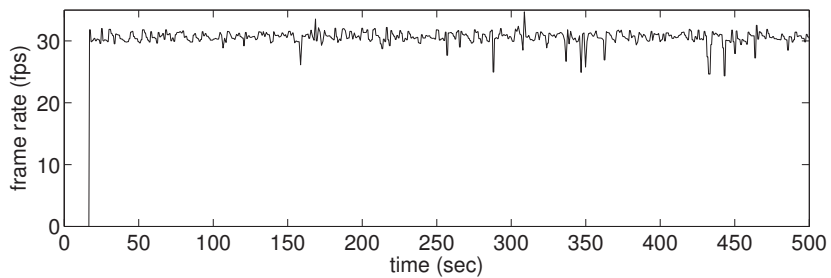

(c) Frame rate $f(t)$

Figure 11: QAC response to a square-wave available bandwidth with period $200 \mathrm{~s}$

1) when the available bandwidth increases to $A_{M}$ the video level is increased to $l_{3}$, which is less than the maximum video level $l_{4}$, in around $75 \mathrm{~s} ; 2$ ) when bandwidth drops occur the playback is affected by interruptions as it can be inferred by considering Figure 12(b) and Figure 12(c). In particular, when the first bandwidth drop occurs at $t=200 \mathrm{~s}$, a BUFFER_FAILURE is sent to the server after a delay of roughly $7 \mathrm{~s}$ in order to switch down the video level from $l_{3}$ to $l_{0}$. After that, a switch-down delay $\tau_{s d}$ of 20 s occurs and the video level $l(t)$ is finally switched to $l_{0}$. Thus, the total delay spent to correctly set the video level $l(t)$ to match the new value of the available bandwidth is $38 \mathrm{~s}$. Due to this large delay in setting $l(t)$, the receiver buffer gets empty and the reproduction of the video is blocked for more than 100s. The same situation occurs when the second bandwidth drop occurs. In this case, the total delay spent to correctly set the video level is 26s. Again, 13s after the second bandwidth drop, an interruption in the video reproduction occurs. During the time intervals with bandwidth $A_{M}=4000 \mathrm{kbps}$, we evaluated a low index of efficiency equal to 0.4 , which is less than half the efficiency obtained by QAC in this scenario.

To summarize, this experiment has shown that the pro- 


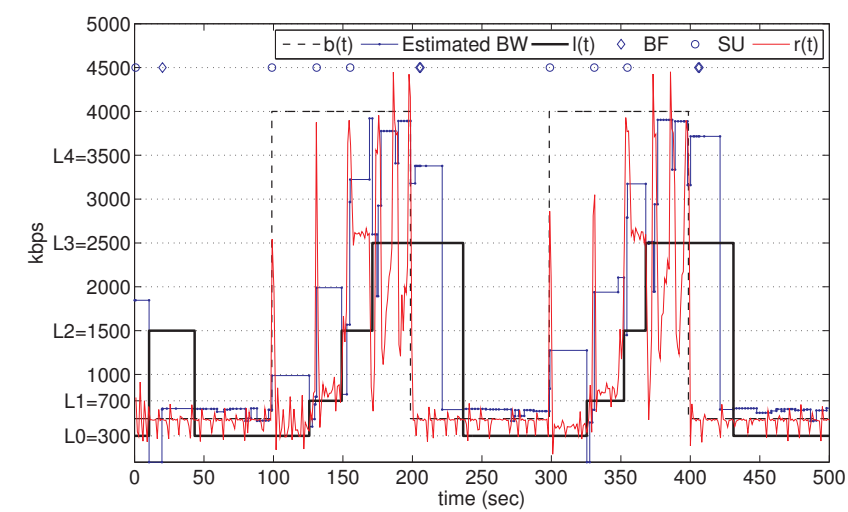

(a) Estimated BW, video level $l(t)$, received rate $r(t)$, BUFFER_FAILURE , and SWITCH_UP events

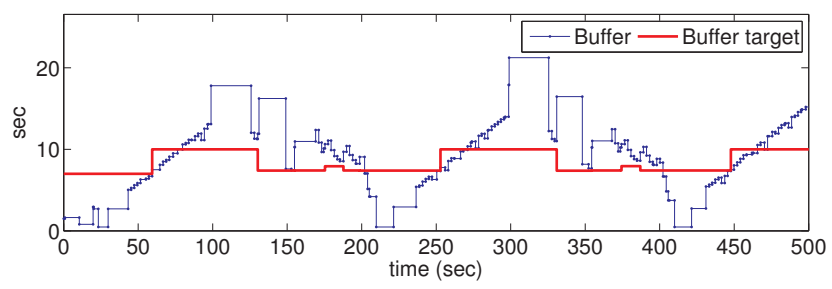

(b) Receiver buffer length and target buffer length

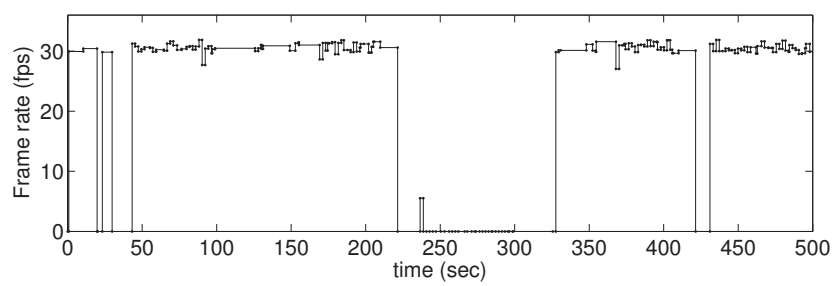

(c) Frame rate $f(t)$

Figure 12: AHDVS response to a square-wave available bandwidth with period $200 \mathrm{~s}$

posed QAC is able to control $l(t)$ to follow step increases and decreases of the available bandwidth always providing the user with a continuous reproduction of the video content at the best QoE. In the case of Akamai HD Video Streaming, when the available bandwidth suddenly shrinks, the video reproduction is affected by interruptions.

\subsection{One concurrent greedy TCP flow}

In this experiment we investigate the performance of the two quality adaptation algorithms when sharing the available bandwidth with one greedy TCP flow, such as in the case of a parallel download session. The available bandwidth has been set to a constant value of $4000 \mathrm{kbps}$, a video streaming session is started at $t=0$, a greedy TCP connection is started at $t=150 \mathrm{~s}$ and it is stopped at $t=360 \mathrm{~s}$.

Figure 13(a) shows the dynamics of the video level $l(t)$ and of the video received rate $r(t)$, whereas Figure 13(b) shows the goodput of the concurrent TCP flow. In the first part of the experiment, for $0<t<150 \mathrm{~s}, l(t)$ quickly matches the available bandwidth obtaining an efficiency $\eta=0.98$. After the greedy TCP flow is started at $t=150$ s the video level $l(t)$ is switched down in about 10s and, since the fair share is

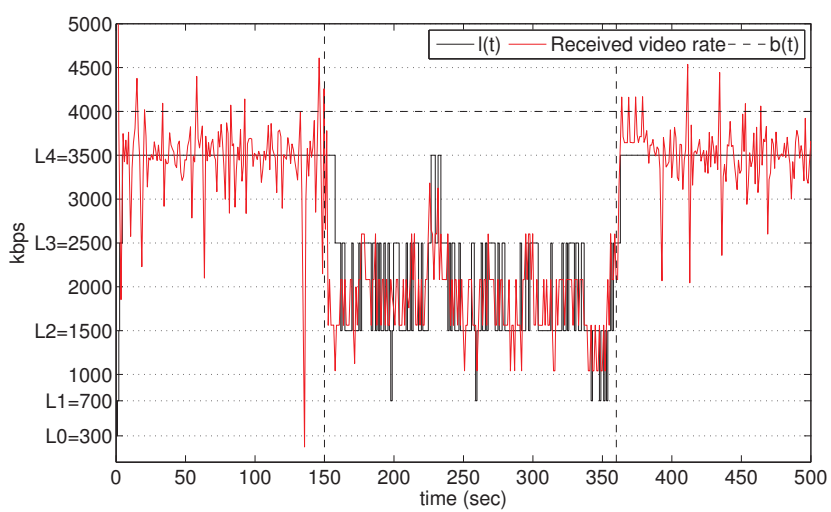

(a) Received rate $r(t)$, video level $l(t)$, and available bandwidth $b(t)$

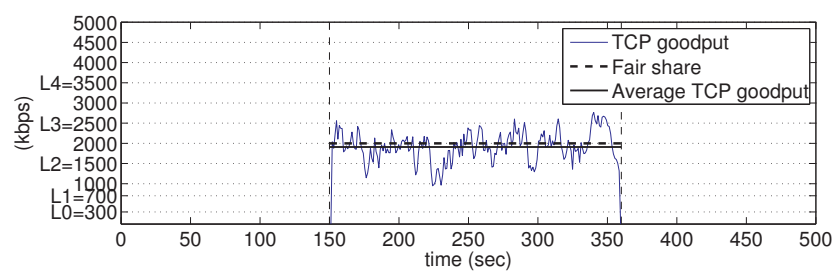

(b) TCP goodput

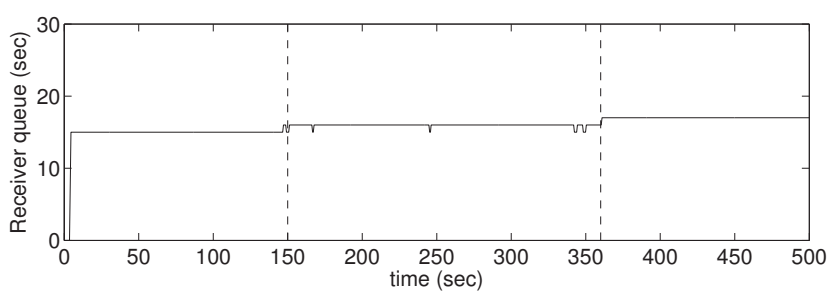

(c) Receiver buffer length

Figure 13: QAC when sharing the bottleneck with one greedy TCP flow

$2000 \mathrm{kbps}, l(t)$ switches between the two closest video levels $l_{2}=1500 \mathrm{kbps}$ and $l_{3}=2500 \mathrm{kbps}$. In this part of the experiment the efficiency is 0.99 and, the average goodput of the greedy TCP flow is $1930 \mathrm{kbps}$ whereas the goodput obtained by QAC flow is $1910 \mathrm{kbps}$ thus indicating that the two flows share the available bandwidth fairly. When the greedy TCP flow is stopped, the video level $l(t)$ is correctly set to the maximum video level $l_{4}$ after a transient of $4 \mathrm{~s}$. In this part of the experiment the efficiency of QAC is 0.99 . Finally, Figure 13(c) shows that the receiver buffer length is always greater than $15 \mathrm{~s}$, meaning that no interruptions occurred during the video reproduction.

Figure 14(a) shows the video level dynamics $l(t)$, the estimated bandwidth and the received video rate $r(t)$ in the case of AHDVS. During the first part of the experiment, i.e. for $t<150 \mathrm{~s}$, apart from a short time interval [6.18,21.93]s during which $l(t)$ is equal to $l_{4}=3500 \mathrm{kbps}$, the video level is set to $l_{3}=2500 \mathrm{kbps}$. The efficiency index $\eta$ in this part of the experiment is 0.74 . When the TCP flow joins the bottleneck, it grabs the fair bandwidth share of $2000 \mathrm{kbps}$. Nevertheless, the estimated bandwidth decreases to the correct value after $9 \mathrm{~s}$. After an additional delay of $8 \mathrm{~s}$, at $t=167 \mathrm{~s}$, a BUFFER_FAILURE command is sent (see Figure 14(a)). The 


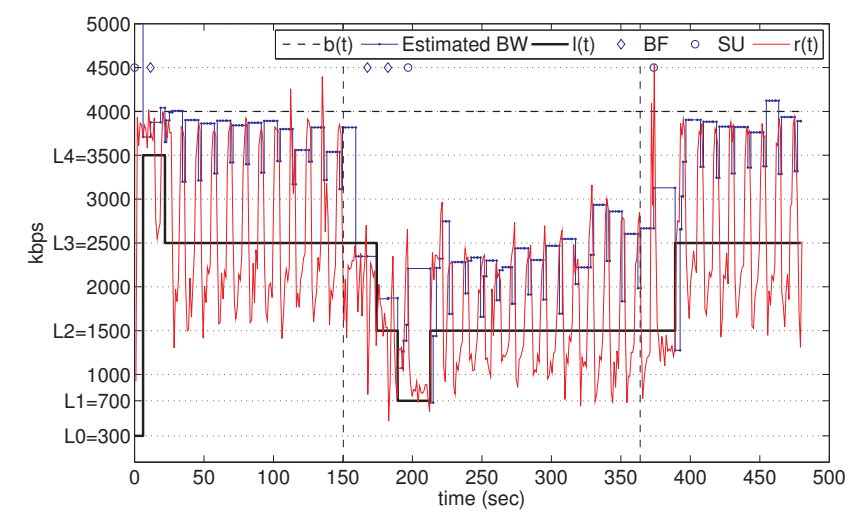

(a) Estimated BW, video level $l(t)$, received rate $r(t)$, BUFFER_FAILURE , and SWITCH_UP events

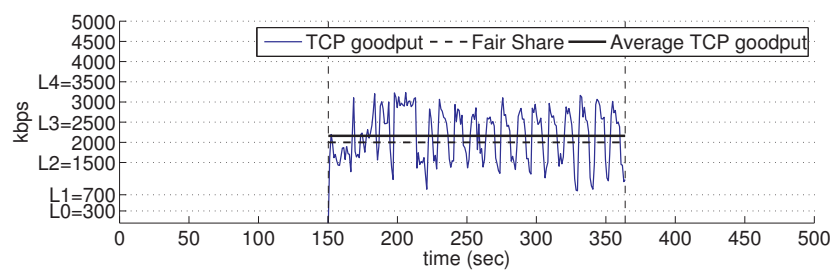

(b) TCP goodput

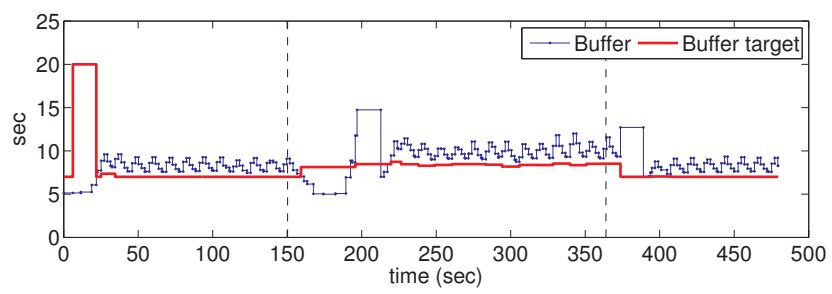

(c) Receiver buffer length and target buffer length

Figure 14: AHDVS when sharing the bottleneck with one greedy TCP flow

video level is shrunk to the suitable value $l_{2}=1500 \mathrm{kbps}$ after a total delay of $24 \mathrm{~s}$. In this case, this actuation delay does not affect the video reproduction as it can be inferred by considering the receiver buffer dynamics shown in Figure 14(c). However, Figure 14(a) shows that $l(t)$ is further decreased to $l_{1}=700 \mathrm{kbps}$ and it is set to steady state value of $l_{2}$ at $t=212 \mathrm{~s}$. Thus, the transient time spent to reach the steady state is $62 \mathrm{~s}$. In this part of the experiment, the efficiency index is equal to 0.76 , the average goodput of the greedy TCP flow is $2170 \mathrm{kbps}$, whereas the goodput obtained by Akamai flow is $1643 \mathrm{kbps}$ indicating that the available bandwidth is underutilized. In the third part of the experiment, after the TCP flow leaves the bottleneck at time $t=360 \mathrm{~s}$, the level is switched up to $l_{3}=2500 \mathrm{kbps}$ with a delay of $26 \mathrm{~s}$. In this part of the experiment the efficiency is 0.69. Finally, by considering Figure 14(b), we can observe that the "on-off" dynamics of the sending rate provided by AHDVS affects the dynamics of the TCP received rate that shows remarkable oscillations.

\subsection{Two concurrent video streaming sessions}

In this scenario we evaluate the behaviour of two video streams that share the same bottleneck whose available band-

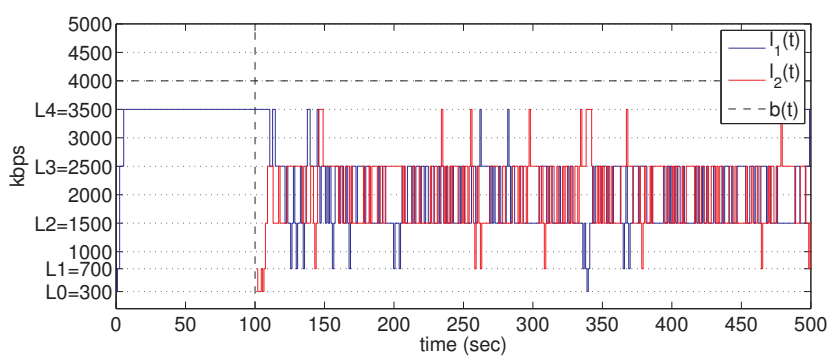

(a) Received video levels $l_{1}(t), l_{2}(t)$

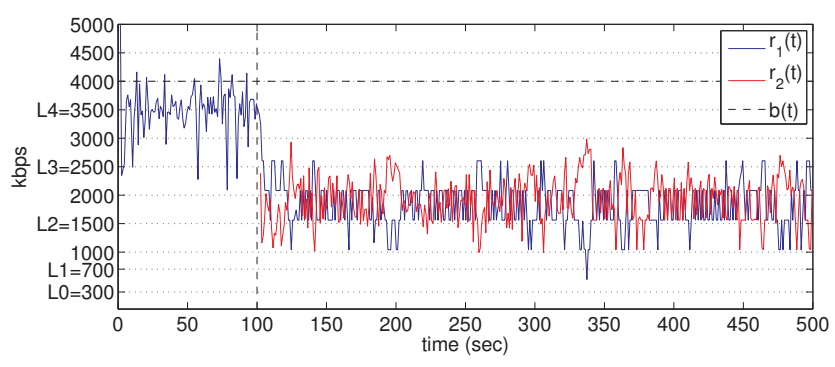

(b) Received rates $r_{1}(t)$ and $r_{2}(t)$

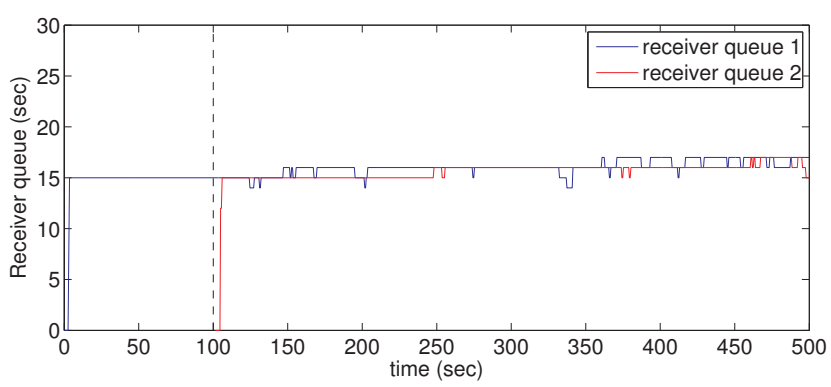

(c) Receiver queue of the two concurrent video flows

Figure 15: Two QAC adaptive video streaming flows sharing a bottleneck

width has been set to $4000 \mathrm{kbps}$. The first video streaming session is started at $t=0$ and after 100 s a second video flow is started. This experiment is aimed at assessing to what extent two competing flows are able to share in a fair way the bottleneck. In this experiment the fair share is equal to 2000 kbps.

Figure 15(a) shows the dynamics of the video levels $l_{1}(t)$ and $l_{2}(t)$ of the first and the second video flow controlled by QAC. In the first part of the experiment, the first flow behaves as already shown in the other experiments quickly setting $l_{1}(t)$ to the maximum video level $l_{4}$. When the second video flow joins the bottleneck at $t=100 \mathrm{~s}$, the video level $l_{1}(t)$ is correctly shrunk to let the second video flow obtain its fair share. After a transient time of $8 \mathrm{~s}$ the two video levels $l_{1}(t)$ and $l_{2}(t)$ start to switch between the two video levels, $l_{2}=1500 \mathrm{kbps}$ and $l_{3}=2500 \mathrm{kbps}$, that are closest to the fair share which is $2000 \mathrm{kbps}$.

Figure 16(a) shows the dynamics of the two video levels in the case of AHDVS. The figure shows that, when the second flow joins the bottleneck, it takes 210 s for the video level $l_{1}(t)$ to be set to the correct value $l_{2}=1500 \mathrm{kbps}$. Thus, during this transient the first video flow experiences a higher video level with respect to the second video flow, 


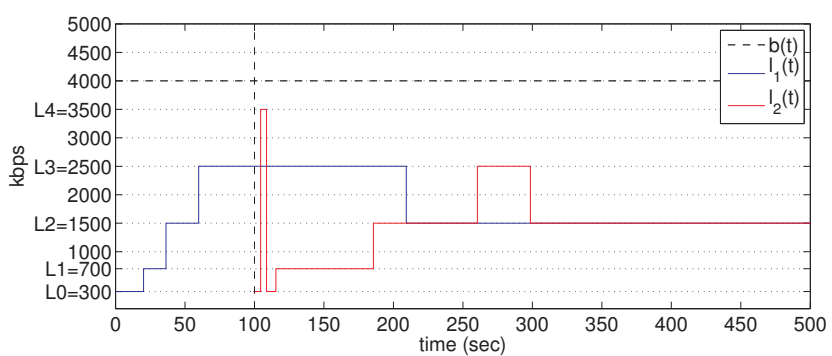

(a) Received video levels $l_{1}(t), l_{2}(t)$

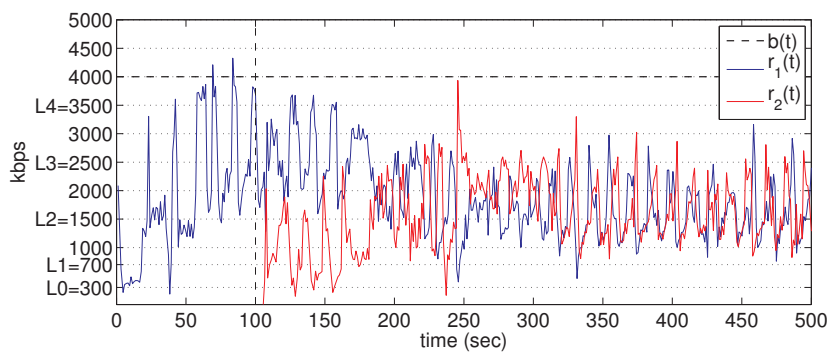

(b) Received video rates $r_{1}(t)$ and $r_{2}(t)$

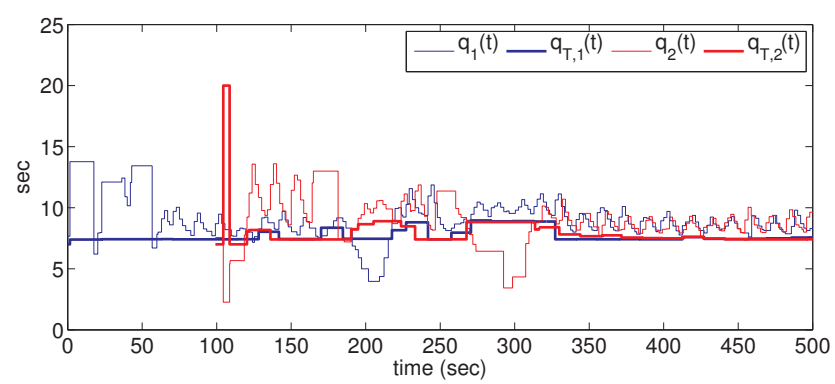

(c) Receiver buffer lengths $q_{1}(t)$ and $q_{2}(t)$ and target buffer lengths $q_{T, 1}(t), q_{T, 2}(t)$

Figure 16: Two AHDVS flows sharing a bottleneck

indicating that the controller is not able to provide the same QoE to all the users sharing a bottleneck.

Finally, Table 3 collects the average goodputs $g_{1}$ and $g_{2}$ obtained for $t>100$ s by the first and the second flow respectively for both QAC and AHDVS streaming systems. The average channel utilization, computed as $U=\left(g_{1}+\right.$ $\left.g_{2}\right) / 4000$ kbps, obtained by QAC results $10 \%$ higer wrt the one obtained by AHDVS.

\section{CONCLUSIONS}

In this paper we have presented a Quality Adaptation Controller (QAC) for a stream-switching adaptive live video streaming system designed by using feedback control theory. Moreover, we have provided a characterization of the adaptation algorithm employed by Akamai High Definition Video Server which also implements a stream-switching system.

The main results of the paper are the following: 1) QAC is able to control the video level $l(t)$ to match the available bandwidth $b(t)$ with a transient time that is less than $30 \mathrm{~s}$ always providing a continuous video reproduction; 2) the proposed controller is able to share in a fair way the available bandwidth both in the case of a concurrent greedy connection and a concurrent video streaming flow; 3) Akamai underutilizes the available bandwidth due to the conserva-

\begin{tabular}{|c|c|c|c|}
\hline Server & $g_{1}$ & $g_{2}$ & $U$ \\
\hline \hline QAC & 1860 & 1950 & 0.95 \\
\hline AHDVS & 1815 & 1612 & 0.85 \\
\hline
\end{tabular}

Table 3: Goodput $g_{1}$ and $g_{2}$ (kbps) of the two concurrent flows and channel utilization $U$

tiveness of its algorithm based on heuristics; 4) moreover, when abrupt reductions of the available bandwidth occur, the video reproduction is affected by interruptions.

\section{REFERENCES}

[1] Akamai HD Network Demo. http://wwwns.akamai.com/hdnetwork/demo/flash.

[2] Move Networks HD adaptive video streaming. http://www.movenetworkshd.com.

[3] Adobe Systems Inc. Real-Time Messaging Protocol (RTMP) Specification. 2009.

[4] L. De Cicco and S. Mascolo. A Mathematical Model of the Skype VoIP Congestion Control Algorithm. IEEE Trans. on Automatic Control, 55(3):790-795, Mar. 2010.

[5] L. De Cicco and S. Mascolo. An Experimental Investigation of the Akamai Adaptive Video Streaming. In Proc. of USAB 2010, Nov. 4-5, 2010.

[6] G. Franklin, J. Powell, and A. Emami-Naeini. Feedback control of dynamic systems. Addison-Wesley, 1994.

[7] M. Handley, S. Floyd, and J. Pahdye. TCP Friendly Rate Control (TFRC): Protocol Specification. $R F C$ 3448, Proposed Standard, Jan. 2003.

[8] D. Hassoun. Dynamic streaming in flash media server 3.5. Available: http://www.adobe.com/devnet/flashmediaserver/.

[9] C. Krasic, J. Walpole, and W. Feng. Quality-adaptive media streaming by priority drop. In Proc. of $A C M$ NOSSDAV '03, 2003.

[10] R. Kuschnig, I. Kofler, and H. Hellwagner. An evaluation of TCP-based rate-control algorithms for adaptive internet streaming of H. 264/SVC. In Proc. of ACM SIGMM conference on Multimedia systems, pages $157-168,2010$.

[11] R. Pantos and W. May. HTTP Live Streaming. IETF Draft, June 2010.

[12] M. Prangl, I. Kofler, and H. Hellwagner. Towards QoS Improvements of TCP-Based Media Delivery. In Proc. of ICNS '08, pages 188-193, 2008.

[13] S. Mascolo. Congestion control in high-speed communication networks using the Smith principle. Automatica, 35(12):1921-1935, 1999.

[14] H. Schulzrinne, A. Rao, and R. Lanphier. Real Time Streaming Protocol (RTSP). RFC 2326, Standard track, Apr. 1998.

[15] V. Jacobson. Congestion avoidance and control. In Proc. of ACM SIGCOMM '88, pages 314-329, 1988.

[16] B. Wang, J. Kurose, P. Shenoy, and D. Towsley. Multimedia streaming via TCP: An analytic performance study. ACM TOMCCAP, 4(2):1-22, 2008.

[17] A. Zambelli. IIS smooth streaming technical overview. Microsoft Corporation, 2009. 